\title{
220 In the 1987 UICC classification of malignant tumours of the larynx
}

A The posterior commissure is part of the glottis.

B A glottic tumour involving both cords with normal mobility and no extension to other sites is a T1b lesion.

C A tumour limited to the larynx with cord fixation is T3.

D The presence of 2 cervical lymph node metastases makes the patient Stage IV.

E A thyroid cartilage chondrosarcoma which has invaded into the thyroid gland is T4.

\section{Spread of malignant disease of the larynx}

A Tumours of the anterior commissure can extend directly into the petiolus.

B The true vocal cords are virtually devoid of lymph vessels.

C About $40 \%$ of tumours of the false cords and ventricular sinuses have metastasized at the time of diagnosis.

D There is a paucity of transglottic lymph vessels in the vertical plane of the larynx.

E Epiglottic tumours may metastasize to the submandibular and submental nodes. 